\title{
Abelian Kernels of Monoids of Order-preserving Maps and of Some of Its Extensions
}

\section{Manuel Delgado and Vítor H. Fernandes}

Communicated by Benjamin Steinberg

\begin{abstract}
We compute the abelian kernels of some monoids of partial transformations. More precisely, the cases of the symmetric inverse monoid, monoids of order-preserving or order-reversing transformations and monoids of orientationpreserving or orientation-reversing transformations are treated.
\end{abstract}

\section{Introduction}

The notion of kernel of a finite monoid became very popular among Semigroup Theorists through a conjecture known as Rhodes Type II Conjecture which proposed an algorithm to perform its computation. It survived as a conjecture almost twenty years and became a theorem after independent and deep work of Ash [2] and Ribes and Zalesskii [19]. For history, motivation and consequences of the conjecture we refer the reader to [13].

The abelian counterpart of the Rhodes Type II Conjecture was solved by the first author in [4]. The algorithm there obtained to compute the abelian kernel of a finite monoid, although exponential, was optimized and rendered usable in practice in [5]. It has been implemented in the GAP language [21] and is part of a package currently under development.

Some classes of (inverse) monoids of injective partial transformations have been the object of study of the second author: in [10] several structural properties of the monoids $\mathcal{P} \mathcal{O I}_{n}$ of all injective order-preserving partial transformations on a chain with $n$ elements were studied and a presentation for these monoids was given; in [9] a similar study was presented for the monoids $\mathcal{P O P} \mathcal{I}_{n}$ of all injective orientation-preserving partial transformations on a chain with $n$ elements. The second author, in joint work with Gomes and Jesus [12] (see also the survey [11]), considered other extensions of the inverse monoid $\mathcal{P O} \mathcal{I}_{n}$. With particular interest for this paper, are the following inverse monoids: $\mathcal{P O D} \mathcal{I}_{n}$

M. Delgado gratefully acknowledges support of FCT through CMUP and the FCT and POCTI Project POCTI/32817/MAT/2000 which is funded in cooperation with the European Community Fund FEDER. V. H. Fernandes gratefully acknowledges support of FCT and FEDER, within the POCTI project "Fundamental and Applied Algebra" of CAUL. 
consisting of all injective partial transformations on a chain with $n$ elements which are either order-preserving or order-reversing; $\mathcal{P O R} \mathcal{I}_{n}$ consisting of all injective orientation-preserving or orientation-reversing partial transformations on a chain with $n$ elements; and $\mathcal{I}_{n}$, the symmetric inverse monoid on a base set with $n$ elements.

We have described in [6] the abelian kernel of two of these classes, namely of the monoids $\mathcal{P O \mathcal { I } _ { n }}$ and of the monoids $\mathcal{P O P \mathcal { I } _ { n }}$, and extracted as a consequence a counterexample for a quite natural conjecture: we have deduced that

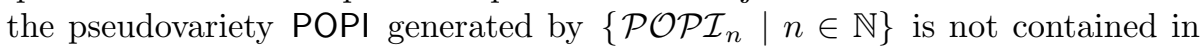
the Mal'cev product of $\mathrm{POI}$, the pseudovariety generated by $\left\{\mathcal{P O} \mathcal{I}_{n} \mid n \in \mathbb{N}\right\}$, and $A b$, the pseudovariety of all finite abelian groups. In some sense, we pursue here this work, computing now the abelian kernels of the monoids $\mathcal{P O D \mathcal { I } _ { n }}$, $\mathcal{P O R} \mathcal{I}_{n}$ and $\mathcal{I}_{n}$.

The techniques used here, although more elaborated in some cases, are similar to those of [6]. Furthermore, several results of [6] will be used.

The first two sections are mostly devoted to background and motivation. The third section contains some results of general use. The remaining sections include the descriptions of the abelian kernels of the monoids $\mathcal{P O D \mathcal { I } _ { n }}, \mathcal{P O R} \mathcal{I}_{n}$ and $\mathcal{I}_{n}$.

\section{The monoids $\mathcal{I}_{n}, \mathcal{P O D I} \mathcal{I}_{n}$ and $\mathcal{P O R} \mathcal{I}_{n}$}

In this section we give some background on the inverse monoids whose abelian kernel will be described. The facts recalled will be used in this description.

For general background on Green relations and inverse semigroups, we refer the reader to Howie's book [14].

Let $M$ be a monoid. Recall the definition of the quasi-order $\leq \mathscr{J}$ associated to the Green relation $\mathscr{J}$ (which for a finite monoid coincides with the Green relation $\mathscr{D})$. For all $u, v \in M$,

$$
u \leq \mathscr{J} v \text { if and only if } M u M \subseteq M v M .
$$

Notice that, for every $u, v \in M, u \mathscr{J} v$ if and only if $u \leq \mathscr{J} v$ and $v \leq \mathscr{J} u$. Denote by $J_{u}$ the $\mathscr{J}$-class of the element $u \in M$. As usual, a partial order relation $\leq \mathscr{J}$ is defined on the set $M / \mathscr{J}$ by setting, for all $u, v \in M, J_{u} \leq \mathscr{J}$ $J_{v}$ if and only if $u \leq \mathscr{J} v$. Given $u, v \in M$, we write $u<\mathscr{J} v$ and $J_{u}<\mathscr{J} J_{v}$ if and only if $u \leq \mathscr{J} v$ and $(u, v) \notin \mathscr{J}$.

Now, let $R$ be a binary relation on a monoid $M$. Let $u, v \in M$. We say that $u$ is connected to $v$ by an elementary $R$-transition if

$$
u=x a y \text { and } v=x b y \text {, }
$$

for some $x, y \in M$, where either $(a, b)$ or $(b, a)$ belongs to $R$.

For a finite alphabet $A$, denote by $A^{*}$ the free monoid generated by $A$. A monoid presentation is an ordered pair $\langle A \mid R\rangle$, where $A$ is an alphabet and $R$ is a subset of $A^{*} \times A^{*}$. An element $(u, v)$ of $R$ is called a relation and is usually 
represented by $u=v$. A monoid $M$ is said to be defined by the presentation $\langle A \mid R\rangle$ if $M$ is isomorphic to $A^{*} / \rho_{R}$, where $\rho_{R}$ denotes the congruence generated by $R$. Throughout this paper the canonical homomorphism from $A^{*}$ onto $M$ will be denoted by $\varphi$.

Notice that, given words $u, v \in A^{*}$, we have $(u, v) \in \rho_{R}$ if and only if there exists a finite sequence $u=u_{0}, u_{1}, \ldots, u_{k}=v(k \geq 0)$ of words such that, for all $0 \leq i \leq k-1, u_{i}$ is connected to $u_{i+1}$ by an elementary $R$-transition.

Let $u \in A^{*}$. We say that $R$ can be applied to $u$ if there exists $v \in A^{*}$ and an elementary $R$-transition connecting $u$ to $v$.

To avoid ambiguities, from now on we take $n \geq 4$. Notice that, for $n \leq 3$, the abelian kernel of the finitely many semigroups in consideration can be easily computed using the first author implementation in GAP [21] of the algorithm presented in [4].

\subsection{The monoid $\mathcal{I}_{n}$}

We begin by recalling some well known facts on the symmetric inverse monoid $\mathcal{I}_{n}$ on a base set with $n$ elements, i.e. the inverse monoid (under composition) of all injective partial transformations on a set with $n$ elements.

Let $\mathcal{S}_{n}$ be the symmetric group on a base set with $n$ elements. Consider the transposition $a=(12)$ and the $n$-cycle $g=(12 \cdots n)$ of $\mathcal{S}_{n}$. Then, it is well known that $\{a, g\}$ is a set of generators of $\mathcal{S}_{n}$ and, from a group presentation due to Moore [16], one can easily deduce [11] the following monoid presentation for $\mathcal{S}_{n}$, in terms of these generators:

$$
\left\langle a, g \mid a^{2}=g^{n}=(g a)^{n-1}=\left(a g^{n-1} a g\right)^{3}=\left(a g^{n-j} a g^{j}\right)^{2}=1 \quad(2 \leq j \leq n-2)\right\rangle .
$$

Notice that $\mathcal{S}_{n}$ is the group of units of the monoid $\mathcal{I}_{n}$ and that two elements of $\mathcal{I}_{n}$ are $\mathscr{R}$-related or $\mathscr{L}$-related if they have the same domain or the same image, respectively. Moreover, given $s, t \in \mathcal{I}_{n}$, we have $s \leq \mathscr{J} t$ if and only if $|\operatorname{Im}(s)| \leq|\operatorname{Im}(t)|$. Hence, for $k \in\{0,1, \ldots, n\}$, being $J_{k}=\left\{s \in \mathcal{I}_{n} \mid\right.$ $|\operatorname{Im}(s)|=k\}$, we have

$$
\mathcal{I}_{n} / \mathscr{J}=\left\{J_{0}<\mathscr{J} J_{1}<\mathscr{J} \cdots<\mathscr{J} J_{n}\right\}
$$

Since $\left|J_{k}\right|=\left(\begin{array}{l}n \\ k\end{array}\right)^{2} k$ !, for $k \in\{0,1, \ldots, n\}$, it follows that $\mathcal{I}_{n}$ has $\sum_{k=0}^{n}\left(\begin{array}{l}n \\ k\end{array}\right)^{2} k$ ! elements. Observe that $J_{n}=\mathcal{S}_{n}$. Moreover, the maximal subgroups of $\mathcal{I}_{n}$ contained in $J_{k}$ are isomorphic to $\mathcal{S}_{k}$, for $1 \leq k \leq n$. We obtain a generating set of $\mathcal{I}_{n}$, with three elements, by joining to the permutations $a$ and $g$ any injective partial transformation of rank $n-1$. For instance, if

$$
c=\left(\begin{array}{cccc}
2 & 3 & \cdots & n \\
2 & 3 & \cdots & n
\end{array}\right)
$$

then the set $\{a, g, c\}$ generates the monoid $\mathcal{I}_{n}$. 
Combining the above monoid presentation of $\mathcal{S}_{n}$ with the Popova presentation of $\mathcal{I}_{n}$ [18], one can deduce [11] the following presentation of $\mathcal{I}_{n}$, in terms of the generators $a, g$ and $c$ :

$$
\begin{aligned}
\langle a, g, c| & a^{2}=g^{n}=(g a)^{n-1}=\left(a g^{n-1} a g\right)^{3}=\left(a g^{n-j} a g^{j}\right)^{2}=1(2 \leq j \leq n-2), \\
& \left.g^{n-1} a g c g^{n-1} a g=g a c a g^{n-1}=c=c^{2},(c a)^{2}=c a c=(a c)^{2}\right\rangle .
\end{aligned}
$$

\subsection{The monoid $\mathcal{P O D} \mathcal{I}_{n}$}

Now, we focus our attention on the monoid $\mathcal{P O D} \mathcal{I}_{n}$. We begin by making precise some notions. A partial transformation $s$ of a chain with $n$ elements is called order-preserving (respectively, order-reversing) if, for all $x, y \in \operatorname{Dom}(s)$, $x \leq y$ implies $x s \leq y s$ (respectively, $x s \geq y s$ ). Clearly, the product of two order-preserving transformations or two order-reversing transformations is an order-preserving transformation and the product of an order-preserving transformation by an order-reversing transformation, or vice-versa, is an orderreversing transformation. Recall that $\mathcal{P O \mathcal { I } _ { n }}$ denotes the inverse submonoid of $\mathcal{I}_{n}$ of all order-preserving transformations and $\mathcal{P O D} \mathcal{I}_{n}$ denotes the inverse submonoid of $\mathcal{I}_{n}$ whose elements are all order-preserving or order-reversing transformations. Notice that, by definition, $\mathcal{P O \mathcal { I } _ { n }}$ is a submonoid of $\mathcal{P O D \mathcal { I } _ { n }}$.

Next, we exhibit some properties as well as a presentation of the monoid $\mathcal{P O D} \mathcal{I}_{n}$, which were given by the second author, Gomes and Jesus in [12].

The second author proved in [8] that $\mid \mathcal{P O \mathcal { I } _ { n } |}=\left(\begin{array}{c}2 n \\ n\end{array}\right)$ and, from this result, it was deduced that $\left|\mathcal{P O D} \mathcal{I}_{n}\right|=2\left(\begin{array}{c}2 n \\ n\end{array}\right)-n^{2}-1$, using the fact that the $\mathscr{H}$-class of an element $s \in \mathcal{P O D \mathcal { I } _ { n }}$ has exactly two elements (an orderpreserving one and another being order-reversing), unless the rank of $s$ is one or zero, in which case its $\mathscr{H}$-class is trivial. As for $\mathcal{I}_{n}$, given $s, t \in \mathcal{P O D} \mathcal{I}_{n}$, we have $s \leq \mathscr{J} t$ if and only if $|\operatorname{Im}(s)| \leq|\operatorname{Im}(t)|$, whence

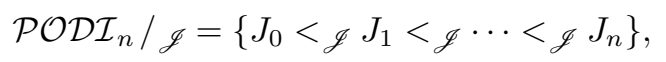

where $J_{k}=\left\{s \in \mathcal{P} \mathcal{O D} \mathcal{I}_{n}|\operatorname{Im}(s)|=k\right\}$, for $k \in\{0,1, \ldots, n\}$.

Now, we recall the presentation of the monoid $\mathcal{P O D} \mathcal{I}_{n}$ exhibited in [12], in terms of $n+1$ generators, although this monoid has smaller rank. In fact, the monoid $\mathcal{P O D} \mathcal{I}_{n}$ has rank $\left\lceil\frac{n}{2}\right\rceil+1$, the least integer greater than or equal to $\frac{n}{2}+1$.

Let $x_{0}, x_{1}, \ldots, x_{n-1}, h$ be $n+1$ letters and let $Y=\left\{x_{0}, x_{1}, \ldots, x_{n-1}, h\right\}$.

In the expressions of the relations below, the following convention will be adopted: given $i, j \in\{0, \ldots, n-1\}$, if $i \leq j$ the expression $x_{i} \cdots x_{j}$ represents the word of length $j-i+1$ such that the letter in the position $p \in\{1, \ldots, j-i+1\}$ is $x_{i+p-1}$ (i.e. the indexes of the letters are ordered in the usual way and are consecutive), and if $j<i$ the expression $x_{i} \cdots x_{j}$ represents the empty word. For example, the expression $x_{3} \cdots x_{2}$ denotes the empty word and not the word $x_{3} x_{2}$.

Consider the following set of monoid relations:

$\left(R_{1}\right) x_{i} x_{0}=x_{0} x_{i+1}, 1 \leq i \leq n-2 ;$ 
$\left(R_{2}\right) x_{j} x_{i}=x_{i} x_{j}, 2 \leq i+1<j \leq n-1$;

$\left(R_{3}\right) x_{0}^{2} x_{1}=x_{0}^{2}=x_{n-1} x_{0}^{2}$;

$\left(R_{4}\right) x_{i+1} x_{i} x_{i+1}=x_{i+1} x_{i}=x_{i} x_{i+1} x_{i}, 1 \leq i \leq n-2 ;$

$\left(R_{5}\right) x_{i} x_{i+1} \cdots x_{n-1} x_{0} x_{1} \cdots x_{i-1} x_{i}=x_{i}, 0 \leq i \leq n-1$;

( $\left.R_{6}\right) x_{i+1} \cdots x_{n-1} x_{0} x_{1} \cdots x_{i-1} x_{i}^{2}=x_{i}^{2}, 1 \leq i \leq n-1$;

$\left(N R_{0}\right) h^{2}=1$

$\left(N R_{1}\right) h x_{0}=x_{1} \cdots x_{n-1} h$ and, for $1 \leq i \leq n-1$,

$$
h x_{i}=x_{n-i+1} \cdots x_{n-1} x_{0} x_{1} \cdots x_{n-i-1} h ;
$$

$\left(N R_{2}\right) x_{0}^{n-1} h=x_{n-1} \cdots x_{3} x_{2}^{2}$.

Let $R=R_{1} \cup R_{2} \cup R_{3} \cup R_{4} \cup R_{5} \cup R_{6} \cup N R_{0} \cup N R_{1} \cup N R_{2}$. Then $\langle Y \mid R\rangle$ is a presentation of the monoid $\mathcal{P O D \mathcal { I } _ { n }}$. We notice that this presentation of $\mathcal{P O D I} \mathcal{I}_{n}$ is based on the presentation of $\mathcal{P O \mathcal { I } _ { n }}$,

$$
\left\langle x_{0}, x_{1}, \ldots, x_{n-1} \mid R_{1} \cup R_{2} \cup R_{3} \cup R_{4} \cup R_{5} \cup R_{6}\right\rangle,
$$

given by the second author in [10]. Also, notice that the $(n+1)^{t h}$ generator $h$ of $X$ corresponds to the permutation of $\mathcal{P O D} \mathcal{I}_{n}$ that reverts the order:

$$
\left(\begin{array}{ccccc}
1 & 2 & \cdots & n-1 & n \\
n & n-1 & \cdots & 2 & 1
\end{array}\right)
$$

The set $\bar{W}_{n-1}$ of canonical forms, considered in [12], corresponding to the elements of the $\mathscr{J}$-class $J_{n-1}$ of $\mathcal{P O D \mathcal { I } _ { n }}$ is defined as follows: being $z=x_{1} \cdots x_{n-1}$ and

$$
W_{n-1}=\left\{u x_{0} v \mid u \text { is a suffix of } z \text { and } v \text { is a prefix of } z\right\},
$$

then

$$
\bar{W}_{n-1}=W_{n-1} \cup\left\{w h \mid w \in W_{n-1}\right\} .
$$

Notice that $W_{n-1}$ is the set of canonical forms, considered by the second author in [10], corresponding to the elements of rank $n-1$ of $\mathcal{P O \mathcal { I } _ { n }}$.

\subsection{The monoid $\mathcal{P O R} \mathcal{I}_{n}$}

Let $c=\left(c_{1}, c_{2}, \ldots, c_{t}\right)$ be a sequence of $t(t \geq 0)$ elements from (for instance) the chain $\{1<2<\cdots<n\}$. We say that $c$ is cyclic (respectively, anticyclic) if there exists no more than one index $i \in\{1, \ldots, t\}$ such that $c_{i}>c_{i+1}$ (respectively, $c_{i}<c_{i+1}$ ), where $c_{t+1}=c_{1}$. Then, given a partial transformation $s$ on the chain $\{1<2<\cdots<n\}$ such that $\operatorname{Dom}(s)=\left\{a_{1}, \ldots, a_{t}\right\}$, 
with $t \geq 0$ and $a_{1}<\cdots<a_{t}$, we say that $s$ is an orientation-preserving (respectively, orientation-reversing) transformation if the sequence of its images $\left(a_{1} s, \ldots, a_{t} s\right)$ is cyclic (respectively, anti-cyclic). The notion of an orientationpreserving transformation was introduced by Catarino and Higgins [3] and also by McAlister [15]. As in the order case, the product of two orientationpreserving transformations or of two orientation-reversing transformations is an orientation-preserving transformation and the product of an orientationpreserving transformation by an orientation-reversing transformation, or viceversa, is an orientation-reversing transformation. We denote by $\mathcal{P O P} \mathcal{I}_{n}$ the inverse submonoid of $\mathcal{I}_{n}$ of all orientation-preserving transformations and by $\mathcal{P O R} \mathcal{I}_{n}$ the inverse submonoid of $\mathcal{I}_{n}$ of all orientation-preserving transformations together with all orientation-reversing transformations.

Now we recall some results on the monoids $\mathcal{P O P \mathcal { I } _ { n }}$.

In [9] the second author showed that $\mathcal{P O P} \mathcal{I}_{n}$ has $1+\frac{n}{2}\left(\begin{array}{c}2 n \\ n\end{array}\right)$ elements and that adding to $\mathcal{P O \mathcal { I } _ { n }}$ the permutation $g=(12 \cdots n)$ we obtain a generating set of $\mathcal{P O P \mathcal { I } _ { n }}$. In fact, in the same paper it was also shown that $\mathcal{P O P} \mathcal{I}_{n}$, as a monoid (and as a semigroup), has rank two (a certain element of $\mathcal{P O \mathcal { I } _ { n }}$ with rank $n-1$ together with $g$ suffices to generate $\left.\mathcal{P} \mathcal{O P} \mathcal{I}_{n}\right)$. Also in [9] a presentation of $\mathcal{P O P \mathcal { I } _ { n }}$ was given. Next, we recall it.

Let $x_{0}, x_{1}, \ldots, x_{n-1}, g$ be $n+1$ letters and let $X=\left\{x_{0}, x_{1}, \ldots, x_{n-1}, g\right\}$. Consider the following set of monoid relations:

$$
\begin{aligned}
& \left(R_{1}\right) x_{i} x_{0}=x_{0} x_{i+1}, 1 \leq i \leq n-2 ; \\
& \left(R_{2}\right) x_{j} x_{i}=x_{i} x_{j}, 2 \leq i+1<j \leq n-1 ; \\
& \left(R_{3}\right) x_{0}^{2} x_{1}=x_{0}^{2}=x_{n-1} x_{0}^{2} ; \\
& \left(R_{4}\right) x_{i+1} x_{i} x_{i+1}=x_{i+1} x_{i}=x_{i} x_{i+1} x_{i}, 1 \leq i \leq n-2 ; \\
& \left(R_{5}\right) x_{i} x_{i+1} \cdots x_{n-1} x_{0} x_{1} \cdots x_{i-1} x_{i}=x_{i}, 0 \leq i \leq n-1 ; \\
& \left(R_{6}\right) x_{i+1} \cdots x_{n-1} x_{0} x_{1} \cdots x_{i-1} x_{i}^{2}=x_{i}^{2}, 1 \leq i \leq n-1 ; \\
& \left(R_{7}\right) g x_{i}=x_{i+1} g, 1 \leq i \leq n-2 ; \\
& \left(R_{8}\right) g x_{0} x_{1}=x_{1} \text { and } x_{n-1} x_{0} g=x_{n-1} ; \\
& \left(R_{9}\right) g^{n}=1 .
\end{aligned}
$$

With $R=R_{1} \cup R_{2} \cup R_{3} \cup R_{4} \cup R_{5} \cup R_{6} \cup R_{7} \cup R_{8} \cup R_{9}$, and based on the presentation of $\mathcal{P O \mathcal { I } _ { n }}$ recalled above, it was proved in [9] that $\langle X \mid R\rangle$ is a presentation for $\mathcal{P O P} \mathcal{I}_{n}$.

Notice that, also in [9] a presentation of $\mathcal{P O P} \mathcal{I}_{n}$ on two generators (with the same number of relations) was given. This presentation was refined by the second author, Gomes and Jesus in [12], by eliminating a large number of superfluous relations. We do not present these results here since they are not relevant for our purposes. 
Now, we concentrate ourselves on the monoid $\mathcal{P O R} \mathcal{I}_{n}$. This monoid was also studied in [12] by the second author, Gomes and Jesus.

As for monoids considered previously, given $s, t \in \mathcal{P O R} \mathcal{I}_{n}$, we have $s \leq \mathscr{J} t$ if and only if $|\operatorname{Im}(s)| \leq|\operatorname{Im}(t)|$. Moreover, if $|\operatorname{Im}(s)|=k \geq 3$ then $H_{s}$, the $\mathscr{H}$-class of $s$, has $2 k$ elements (it is easy to show that, if $H_{s}$ is a maximal subgroup, then it is up to isomorphism the dihedral group

$$
D_{2 k}=\left\langle p, q \mid p^{k}=q^{2}=1, p q=q p^{k-1}\right\rangle
$$

[3] of order $2 k$ ) and if $|\operatorname{Im}(s)|=2$ then $\left|H_{s}\right|=2$, otherwise the $\mathscr{H}$-class of $s$ has just one element. Hence, for $k \in\{0,1, \ldots, n\}$, being $J_{k}=\left\{s \in \mathcal{P O} \mathcal{R} \mathcal{I}_{n} \mid\right.$ $|\operatorname{Im}(s)|=k\}$, we have

$$
\mathcal{P O R} \mathcal{I}_{n} / \mathscr{J}=\left\{J_{0}<\mathscr{J} J_{1}<\mathscr{J} \cdots<\mathscr{J} J_{n}\right\}
$$

and $\left|J_{0}\right|=1,\left|J_{1}\right|=n^{2},\left|J_{2}\right|=2\left(\begin{array}{c}n \\ 2\end{array}\right)^{2}$ and $\left|J_{k}\right|=2 k\left(\begin{array}{c}n \\ k\end{array}\right)^{2}$, for $3 \leq k \leq n$, from what follows that $\left|\mathcal{P O} \mathcal{R} \mathcal{I}_{n}\right|=1+n\left(\begin{array}{c}2 n \\ n\end{array}\right)-\frac{n^{2}}{2}\left(n^{2}-2 n+3\right)$. A set of generators of $\mathcal{P O R \mathcal { I } _ { n }}$ can be obtained by adding the permutation $h$ (defined above) to any set of generators of $\mathcal{P O P} \mathcal{I}_{n}$. In particular, we can find a generating set of $\mathcal{P O R \mathcal { I } _ { n }}$ with just three elements. Notice that in [12] a presentation of $\mathcal{P O R \mathcal { I } _ { n }}$ in terms of three generators was given. This result was accomplished by applying a general technique (described in [12] and also used to obtain the presentation of $\mathcal{P O D} \mathcal{I}_{n}$ already recalled) that consists in considering a presentation of $\mathcal{P O P} \mathcal{I}_{n}$ (that one given in [12]) together with a new letter (corresponding to the generator $h$ ) and a new set of three different kinds of relations involving this new letter. See [12] for details.

The same technique, by mimicking exactly the proof given in [12], allows us to state another presentation of $\mathcal{P O R} \mathcal{I}_{n}$ (more convenient for this paper) this time based on the presentation of $\mathcal{P O P} \mathcal{I}_{n}$ on $n+1$ generators recalled above.

So, let us consider $n+2$ letters $x_{0}, x_{1}, \ldots, x_{n-1}, g, h$ and the set $R$ of monoid relations on the letters $x_{0}, x_{1}, \ldots, x_{n-1}, g$ defined above. Also, consider the following relations:

$\left(N R_{0}\right) h^{2}=1$

$\left(N R_{1}\right) h g=g^{n-1} h, \quad h x_{0}=x_{1} \cdots x_{n-1} h$ and $h x_{i}=x_{n-i+1} \cdots x_{n-1} x_{0} x_{1} \cdots$ $x_{n-i-1} h$, for $1 \leq i \leq n-1$;

$\left(N R_{2}\right)\left(g^{n-1}\left(x_{1} g\right)^{n-1}\right)^{n-2} h=\left(g^{n-1}\left(x_{1} g\right)^{n-1}\right)^{n-2}\left(\left(x_{1} g\right)^{n-2} g^{2}\right)^{n-2} g^{n-1}$.

Let $\bar{R}=R \cup N R_{0} \cup N R_{1} \cup N R_{2}$ and $\bar{X}=\left\{x_{0}, x_{1}, \ldots, x_{n-1}, g, h\right\}$. Then, we have:

Theorem 1.1. The monoid $\mathcal{P O R \mathcal { I } _ { n }}$ is defined by the presentation $\langle\bar{X} \mid \bar{R}\rangle$. 
Observe that the set $\bar{W}_{n-1}$ of canonical forms, considered in [12], corresponding to the elements of the $\mathscr{J}$-class $J_{n-1}$ of $\mathcal{P O R} \mathcal{I}_{n}$ is defined as follows: being $z=x_{1} \cdots x_{n-1}$ and

$$
\begin{aligned}
W_{n-1}=\left\{g^{k} u x_{0} v \mid u \text { is a nonempty suffix of } z, v \text { is a prefix of } z\right. \\
\text { and } 0 \leq k \leq n-1\}
\end{aligned}
$$

then

$$
\bar{W}_{n-1}=W_{n-1} \cup\left\{w h \mid w \in W_{n-1}\right\} .
$$

Notice that $W_{n-1}$ is the set of canonical forms, considered by the second author in [9], corresponding to the elements of rank $n-1$ of $\mathcal{P O P \mathcal { I }}$.

Also, notice that, as a word of the form $x_{i} x_{j}$, with $0 \leq i, j \leq n-1$ and $j \neq(i+1) \bmod n$, corresponds to an element of $\mathcal{P O}_{n}$ of rank $n-2$ (see [10]) and the words of the relation $N R_{2}$ correspond to an element of rank two of $\mathcal{P O R \mathcal { I } _ { n }}$ (in fact, also of $\mathcal{P O P \mathcal { I } _ { n }}$ : see [12]), then the relations of $\bar{R}$ applicable to any word representing an element of the $\mathscr{J}$-class $J_{n-1}$ of $\mathcal{P O R} \mathcal{I}_{n}$ are just those of the set

$$
\tilde{R}=R_{5} \cup R_{7} \cup R_{8} \cup R_{9} \cup N R_{0} \cup N R_{1} .
$$

Our final remark in this section concerns the words of $\bar{W}_{n-1}$ that represent (the $n$ ) idempotents. These are the following:

$$
x_{i} x_{i+1} \cdots x_{n-1} x_{0} x_{1} \cdots x_{i-1}, \quad 1 \leq i \leq n-1,
$$

and $g^{n-1} x_{1} \cdots x_{n-1}$ (which corresponds to the same element of $\mathcal{P O} \mathcal{R} \mathcal{I}_{n}$ that $\left.x_{0} x_{1} \cdots x_{n-1}\right)$.

\section{Abelian kernels}

For basic notions related with rational languages we refer the reader to Pin's book [17]. For profinite topologies and finite semigroups, we refer the reader to Almeida's book [1].

A relational morphism of semigroups $\tau: S \rightarrow T$ is a function from $S$ into $\mathcal{P}(T)$, such that:

(a) For all $s \in S, \tau(s) \neq \emptyset$;

(b) For all $s_{1}, s_{2} \in S, \tau\left(s_{1}\right) \tau\left(s_{2}\right) \subseteq \tau\left(s_{1} s_{2}\right)$.

If $S$ and $T$ are monoids, $\tau$ is called a relational morphism of monoids if it satisfies (a), (b) and

(c) $1 \in \tau(1)$.

A relational morphism $\tau: S \multimap T$ is, in particular, a relation in $S \times T$. Homomorphisms, seen as relations, and inverses of onto homomorphisms are examples of relational morphisms. 
Given a pseudovariety $\mathrm{H}$ of groups, the $\mathrm{H}$-kernel of a finite semigroup $S$ is the subsemigroup $\mathrm{K}_{\mathrm{H}}(\mathrm{S})=\bigcap \tau^{-1}(1)$, with the intersection being taken over all groups $G \in \mathrm{H}$ and all relational morphisms of semigroups $\tau: S \rightarrow G$. Sometimes we refer to the $\mathrm{H}$-kernel simply as relative kernel. When $\mathrm{H}$ is $\mathrm{Ab}$, the pseudovariety of all finite abelian groups, we use the terminology abelian kernel. The H-kernel of a monoid is defined analogously: relational morphisms of monoids instead of relational morphisms of semigroups are taken. The computation of the $\mathrm{H}$-kernel of a finite semigroup can be reduced to the computation of the $\mathrm{H}$-kernel of a finite monoid. In fact, it is straightforward that, for a finite semigroup $S, \mathrm{~K}_{\mathrm{H}}(\mathrm{S})=\mathrm{K}_{\mathrm{H}}\left(\mathrm{S}^{1}\right) \cap \mathrm{S}$, where $S^{1}$ denotes the monoid obtained from $S$ through the adjoining of an identity if $S$ has none and denotes $S$ otherwise.

It follows immediately from the definition that if $N$ is a submonoid of a finite monoid $M$, then $\mathrm{K}_{\mathrm{H}}(\mathrm{N}) \subseteq \mathrm{K}_{\mathrm{H}}(\mathrm{M}) \cap \mathrm{N}$. It is also easy to see that the relative kernel of a finite monoid $M$ is a submonoid of $M$ containing the idempotents. In order to state another useful closure property, we need the definition that follows.

A pair $(s, t)$ of elements of $M$ is said a conjugating pair if sts $=s$ or tst $=t$. A subset $K \subseteq M$ is said to be closed under weak conjugation if whenever $(s, t)$ is a conjugating pair and $u \in K$, then $s u t \in K$.

Proposition 2.1. $\quad$ Let $\mathrm{H}$ be a pseudovariety of groups and let $M$ be a finite monoid. The relative kernel $\mathrm{K}_{\mathrm{H}}(\mathrm{M})$ is a submonoid of $M$ closed under weak conjugation.

The proof of this result is analogous to the corresponding result for the kernel case, which may be found, for example, in [20].

The notion of $\mathrm{H}$-kernel is tightly related to an important operator of pseudovarieties: the Mal'cev product (see [13]). Its definition, when the first factor is a pseudovariety $\mathrm{V}$ of monoids and the second factor is a pseudovariety $\mathrm{H}$ of groups, may be given as follows:

$$
\mathrm{V}(m) \mathrm{H}=\left\{M \in \mathrm{M}: \mathrm{K}_{\mathrm{H}}(\mathrm{M}) \in \mathrm{V}\right\}
$$

Next we collect some results that were first stated in [4] and will be used in the sequel.

The $n$-generated free abelian group $\mathbb{Z}^{n}$ is considered endowed with the profinite group topology, i.e. the least topology rendering continuous all homomorphisms into finite groups.

Proposition 2.2. For $a, b_{1}, \ldots, b_{r} \in \mathbb{N}^{n}$, the closure of the subset $a+b_{1} \mathbb{N}+$ $\cdots+b_{r} \mathbb{N}$ of $\mathbb{Z}^{n}$ is $a+b_{1} \mathbb{Z}+\cdots+b_{r} \mathbb{Z}$.

In order to render our notation more understandable, we will use subscripts in certain components of the elements of $\mathbb{Z}^{n}$. For instance, we write $\left(0, \ldots, 0,1_{(i)}, 0, \ldots, 0\right)$ with the meaning of " $(0, \ldots, 0,1,0, \ldots, 0)$ ( 1 is in the position $i$ )". We adopt the usual notation for the neutral element of an abelian group: $(0, \ldots, 0) \in \mathbb{Z}^{n}$ is simply denoted by 0 . 
Let $A=\left\{a_{1}, \ldots, a_{n}\right\}$ be an alphabet. The canonical homomorphism $\gamma: A^{*} \rightarrow \mathbb{Z}^{n}$ defined by $\gamma\left(a_{i}\right)=\left(0, \ldots, 0,1_{(i)}, 0, \ldots, 0\right)$ will be widely used (for the alphabets in the context). The image under $\gamma$ of a rational language of $A^{*}$ is a rational subset of $\mathbb{N}^{n}\left(\subseteq \mathbb{Z}^{n}\right)$, thus it is a semilinear set, i.e. a finite union of sets of the form $a+b_{1} \mathbb{N}+\cdots+b_{r} \mathbb{N}$. In $[4,5]$ is given an algorithm to compute the image of a rational language of $A^{*}$ by $\gamma$. Of interest for us is the following particular case:

Lemma 2.3. If $u, v_{1}, \ldots, v_{r}, \in A^{*}$, then $\gamma\left(u v_{1}^{*} \cdots v_{r}^{*}\right)=\gamma(u)+\gamma\left(v_{1}\right) \mathbb{N}+$ $\cdots+\gamma\left(v_{r}\right) \mathbb{N}$.

Suppose that $M$ is an $A$-generated finite monoid and let $\varphi: A^{*} \rightarrow M$ be an onto homomorphism. We say that an element $x \in M$ can be represented by a word $w \in A^{*}$ if $\varphi(w)=x$.

Proposition 2.4. An element $x \in M$ belongs to the abelian kernel of $M$ if and only if $0 \in \overline{\gamma\left(\varphi^{-1}(x)\right)}$.

Using the fact that $\varphi^{-1}(x)$ is a rational language (by Kleene's theorem), the observation preceding Lemma 2.3 and Proposition 2.2, we have that $\overline{\gamma\left(\varphi^{-1}(x)\right)}$ is a finite union of sets of the form $a+b_{1} \mathbb{Z}+\cdots+b_{r} \mathbb{Z}$. Thus, when $x \in \mathrm{K}_{\mathrm{Ab}}(M)$, there exist $x_{0}, x_{1}, \ldots, x_{k} \in \mathbb{N}^{n}$ such that $x_{0}+x_{1} \mathbb{N} \cdots+x_{k} \mathbb{N} \subseteq$ $\gamma\left(\varphi^{-1}(x)\right)$ and $0 \in x_{0}+x_{1} \mathbb{Z} \cdots+x_{k} \mathbb{Z}$

The following proposition was proved in a slightly more general form in [4]. See also [6].

Proposition 2.5. $\quad$ The abelian kernel of a finite group $G$ is precisely its derived subgroup $G^{\prime}$.

In particular, the abelian kernel of a finite abelian group is the trivial subgroup. One can easily check [7] that the abelian kernel of the dihedral group $D_{2 n}$ of order $2 n$ is the subgroup

$$
\left\langle g^{2}\right\rangle= \begin{cases}\langle g\rangle, & \text { if } n \text { is odd } \\ \left\{g^{2 i} \mid 0 \leq i \leq \frac{n}{2}\right\}, & \text { if } n \text { is even }\end{cases}
$$

of $D_{2 n}$, where $g$ is the generator of order $n$.

Other examples of abelian kernels are given by the following results:

Theorem 2.6. [6] The abelian kernel of $\mathcal{P O I}_{n}$ consists of all idempotents and all elements of rank less than $n-1$.

Theorem 2.7. [6] The abelian kernel of $\mathcal{P O P} \mathcal{I}_{n}$ consists of all idempotents and all elements of rank less than $n-1$.

Of interest for us is also the following particular case of [6, Lemma 4.8].

Lemma 2.8. Let $G$ be the group of units of a finite monoid $M$. Then $\mathrm{K}_{\mathrm{Ab}}(M) \cap G=G^{\prime}$. 


\section{General results concerning abelian kernels}

Let $n$ be a positive integer and $p_{i}: \mathbb{Z}^{n} \rightarrow \mathbb{Z}$ be the projection onto the $i^{\text {th }}$ component (i.e. for $x=\left(m_{1}, \ldots, m_{n}\right) \in \mathbb{Z}^{n}, p_{i}(x)=m_{i}$ ).

Lemma 3.1. Let $x_{1}, \ldots, x_{k} \in \mathbb{Z}^{n}$ and let $r_{1}, \ldots, r_{k} \in \mathbb{Z}$. Then $p_{i}\left(r_{1} x_{1}+\right.$ $\left.\cdots+r_{k} x_{k}\right)$ and $p_{i}\left(\left|r_{1}\right| x_{1}+\cdots+\left|r_{k}\right| x_{k}\right)$ have the same parity.

Proof. Cleary, it suffices to observe that the sum

$$
p_{i}\left(r_{1} x_{1}+\cdots+r_{k} x_{k}\right)+p_{i}\left(\left|r_{1}\right| x_{1}+\cdots+\left|r_{k}\right| x_{k}\right)
$$

is even. Since

$$
\begin{array}{r}
p_{i}\left(r_{1} x_{1}+\cdots+r_{k} x_{k}\right)+p_{i}\left(\left|r_{1}\right| x_{1}+\cdots+\left|r_{k}\right| x_{k}\right) \\
=\left(r_{1}+\left|r_{1}\right|\right) p_{i}\left(x_{1}\right)+\cdots+\left(r_{k}+\left|r_{k}\right|\right) p_{i}\left(x_{k}\right)
\end{array}
$$

and in this last expression every coefficient is even (it is 0 or $2 r_{i}$ ), the result follows.

Let $B$ be a subset of an alphabet $A$ with $n$ letters. Denote by $|w|_{B}$ the length of a word $w \in A^{*}$ counting only the occurrences of the letters in $B$. If $B$ is singular, say $B=\{a\}$, we adopt the usual notation $|w|_{a}$.

Theorem 3.2. $\quad$ Let $M$ be a finite A-generated monoid. If $x \in \mathrm{K}_{\mathrm{Ab}}(M)$ then, for any letter $a \in A, x$ can be represented by a word $w \in A^{*}$ such that $|w|_{a}$ is even.

Proof. As $x \in \mathrm{K}_{\mathrm{Ab}}(M)$ there exist $x_{0}, x_{1}, \ldots, x_{k} \in \mathbb{N}^{n}$ such that $x_{0}+$ $x_{1} \mathbb{N} \cdots+x_{k} \mathbb{N} \subseteq \gamma\left(\varphi^{-1}(x)\right)$ and $0 \in x_{0}+x_{1} \mathbb{Z} \cdots+x_{k} \mathbb{Z}$, as observed after the statement of Proposition 2.4. Thus there exist $r_{1}, \ldots, r_{k} \in \mathbb{Z}$ such that $0=x_{0}+r_{1} x_{1}+\cdots+r_{k} x_{k}$.

Let $i$ be the position of $a$ in $A$. We have that $p_{i}\left(x_{0}+r_{1} x_{1}+\cdots+r_{k} x_{k}\right)=$ $p_{i}(0)$ is even and so is $p_{i}\left(x_{0}+\left|r_{1}\right| x_{1}+\cdots+\left|r_{k}\right| x_{k}\right)$, by Lemma 3.1. As $x_{0}+\left|r_{1}\right| x_{1}+\cdots+\left|r_{k}\right| x_{k} \in \gamma\left(\varphi^{-1}(x)\right)$, the result follows.

As, for $B=\left\{b_{1}, \ldots, b_{r}\right\} \subseteq A$, we have $|w|_{B}=|w|_{b_{1}}+\cdots+|w|_{b_{r}}$, then we immediately obtain the following corollary:

Corollary 3.3. $\quad$ Let $M$ be a finite A-generated monoid. If $x \in \mathrm{K}_{\mathrm{Ab}}(M)$ then, for any subset $B$ of $A, x$ can be represented by a word $w \in A^{*}$ such that $|w|_{B}$ is even.

Theorem 3.4. Let $T$ be a monoid, let $x_{1}, x_{2}, \ldots, x_{k}, y$ be a set of generators of $T$ such that $y^{2}=1$ and let $S$ be the submonoid of $T$ generated by $x_{1}, x_{2}, \ldots, x_{k}$. If for each $i \in\{1, \ldots, k\}$ there exists $u_{i} \in S$ such that $y x_{i}=u_{i} y$, then $\mathrm{K}_{\mathrm{Ab}}(T) \subseteq S$. 
Proof. Let $x \in \mathrm{K}_{\mathrm{Ab}}(T)$. Then by Theorem 3.2 we can represent $x$ as a word $w \in\left\{x_{1}, x_{2}, \ldots, x_{k}, y\right\}^{*}$ with an even number of occurrences of the generator $y$. Then applying the relations $y x_{i}=u_{i} y, i \in\{1, \ldots, k\}$, from the left hand side to the right hand side of $w$ and the relation $y^{2}=1$ whenever possible, we can represent $x$ without any occurrence of $y$, whence $x \in S$.

Using the properties of the monoids $\mathcal{P O D I} \mathcal{I}_{n}$ and $\mathcal{P O R} \mathcal{I}_{n}$ recalled in Section 1, as an immediate consequence of Theorem 3.4, we have:

Corollary 3.5. One has $\mathrm{K}_{\mathrm{Ab}}\left(\mathcal{P O D} \mathcal{I}_{n}\right) \subset \mathcal{P O \mathcal { I } _ { n }}$.

Corollary 3.6. One has $\mathrm{K}_{\mathrm{Ab}}\left(\mathcal{P O R} \mathcal{I}_{n}\right) \subset \mathcal{P O P \mathcal { I } _ { n }}$.

Let PODI be the pseudovariety generated by $\left\{\mathcal{P O D} \mathcal{I}_{n} \mid n \in \mathbb{N}\right\}$. Using the previous result and the fact that PODI only contains 2 -groups, we obtain the following corollary.

Corollary 3.7. $\quad$ The strict inclusion $\mathrm{PODI} \subset \mathrm{POI} \oplus \mathrm{Ab}$ holds.

Similarly, being PORI be the pseudovariety generated by $\left\{\mathcal{P O} \mathcal{R} \mathcal{I}_{n} \mid n \in\right.$ $\mathbb{N}$ \}, we have:

Corollary 3.8. The inclusion PORI $\subseteq$ POPI $(m)$ Ab holds.

\section{The abelian kernel of $\mathcal{P O D \mathcal { I } _ { n }}$}

Part of the description of the abelian kernel of $\mathcal{P O D \mathcal { I } _ { n }}$ follows immediately from Corollary 3.5, Lemma 2.8 and Theorem 2.6. In fact, as $\mathcal{P O \mathcal { I } _ { n }}$ is a submonoid of $\mathcal{P} \mathcal{O D} \mathcal{I}_{n}$, we have that $\mathrm{K}_{\mathrm{Ab}}\left(\mathcal{P} \mathcal{O} \mathcal{I}_{n}\right) \subseteq \mathrm{K}_{\mathrm{Ab}}\left(\mathcal{P O D} \mathcal{I}_{n}\right) \cap \mathcal{P O} \mathcal{I}_{n}$. Thus, for $k<n-1, \mathrm{~K}_{\mathrm{Ab}}\left(\mathcal{P O O R} \mathcal{I}_{n}\right) \cap J_{k}$ consists of the elements of $J_{k}$ that belong to $\mathcal{P O \mathcal { I } _ { n }}$. On the other hand, since the group of units $J_{n}$ of $\mathcal{P O D} \mathcal{I}_{n}$ is abelian, the only element of $J_{n}$ that belongs to $\mathrm{K}_{\mathrm{Ab}}\left(\mathcal{P O D \mathcal { I } _ { n } )}\right.$ is the neutral element of $\mathcal{P O D \mathcal { I } _ { n }}$. In order to complete the description of the abelian kernel of $\mathcal{P O D \mathcal { I } _ { n }}$, it remains to determine which non idempotents of $\mathcal{P O \mathcal { I } _ { n }} \cap J_{n-1}$ belong to $\mathrm{K}_{\mathrm{Ab}}\left(\mathcal{P O D} \mathcal{I}_{n}\right)$. The parity of $n$ has a great influence, as we shall see.

Let $n$ be an odd integer and let $m=(n+1) / 2$. Define $f_{0}: \mathbb{Z}^{m} \rightarrow \mathbb{Z}^{m}$ by

$$
f_{0}\left(n_{0}, n_{1}, \ldots, n_{m-1}\right)=\left(n_{0}, n_{1}, \ldots, n_{m-1}\right)+(-1,1, \ldots, 1)
$$

and, for $i=1, \ldots, m-1$, define $f_{i}: \mathbb{Z}^{m} \rightarrow \mathbb{Z}^{m}$ by

$$
f_{i}\left(n_{0}, n_{1}, \ldots, n_{m-1}\right)=\left(n_{0}, n_{1}, \ldots, n_{m-1}\right)+\left(1,1, \ldots, 1,0_{(i)}, 1, \ldots, 1\right) .
$$

Lemma 4.1. Let $\left(n_{0}, n_{1}, \ldots, n_{m-1}\right) \in \mathbb{Z}^{m}$. Through successive applications to this element of the functions defined above, one can reach an element of the form $(k, k, \ldots, k)$ in a finite number of steps.

Proof. We start searching for an element of the form $(z, x, \ldots, x)$ without using $f_{0}$. Let $X=\left(n_{0}, n_{1}, \ldots, n_{m-1}\right)$. Define $\|X\|$ as the maximum of the set $\left\{\left|n_{i}-n_{j}\right| \mid 1 \leq i, j \leq m-1\right\}$. 
Suppose that $X$ has not yet the desired form, i.e., $\|X\|>0$. Let $M=$ $\max \left\{n_{1}, \ldots, n_{m-1}\right\}$ and let $\left\{i_{1}, \ldots, i_{r}\right\}$ be the set of indexes such that $n_{i_{j}}=$ $M, j=1, \ldots, r$. Now let us consider the element $X^{\prime}=f_{i_{1}}\left(\ldots\left(f_{i_{r}}(X)\right) \ldots\right)$ and repeat the process with $X=X^{\prime}$ while $\left\|X^{\prime}\right\| \neq 0$. Note that $\left\|X^{\prime}\right\|<\|X\|$, thus this process ends in a finite number of steps.

Now, if the components of $X$ are not yet all equal, then $X$ has the form $(z, x, \ldots, x)$. Several cases may then arise:

Case 1. $z=x+2 s$, with $s$ a positive integer. We apply $f_{0} s$ times and we are done.

Case 2. $z=x+2 s-1$, with $s$ a positive integer. We apply $f_{0} s$ times and then $f_{1} \circ f_{2} \cdots \circ f_{m-1}$.

Case 3. $z=x-s$, with $s$ a positive integer. We apply $f_{1} \circ f_{2} \cdots \circ f_{m-1}$ $s$ times.

Let $x \in \mathcal{P O D I} \mathcal{I}_{n}$. We are aiming to compute a set of words corresponding to $x$, i.e., whose image by $\varphi$ is $x$. Of course, given one such word, any other can be obtained through the application of the relations above.

Observe that, in face of $N R_{0}$, when a word $w$ corresponds to an element $x$ in $\mathcal{P O D I}$, the word $w^{\prime}$ obtained by insertion of $h^{2}$ as a factor at any position of $w$ also corresponds to $x$. This observation allows us to assume that a $h$ is present whenever we need it to use $N R_{1}$.

Suppose that $v$ is a word obtained from $u \in Y^{*}$ through an application of, say, $N R_{1}^{i}$ (the relation from $N R_{1}$ with $h x_{i}$ as left hand side). If $i=0$ the number of occurrences of $x_{0}$ decreases 1 and the number of occurrences of any other letter is raised by 1 . If $i \neq 0$, the number of occurrences of $x_{i}$ and of $x_{n-i}$ stays the same and the number of occurrences of any other letter is raised by 1 .

Suppose now that $u$ is a word of the form $x_{i} \cdots x_{n-1} x_{0} x_{1} \cdots x_{n-i}$ representing an order-preserving transformation of $J_{n-1}$. Recall that we are supposing that $n$ is odd. Thus $i \neq n-i$. Note that the $j^{\text {th }}$ component of $\gamma(u)$ (which corresponds to $\left.x_{j-1}\right)$ equals its $(n-j+1)^{t h}$ component thus we may identify these components, obtaining an element $[\gamma(u)]$ of $\mathbb{Z}^{m}$. Now let us note that $f_{i}([\gamma(u)])=\left[\gamma\left(u^{\prime}\right)\right] \quad(0 \leq i \leq n-1)$, where $u^{\prime}$ is obtained from $u$ by one application of $N R_{1}^{i}$. Thus we can use Lemma 4.1 to produce a word $w$ such that every letter, except possibly $h$, has the same number of occurrences and is such that $\varphi(w)=\varphi(u)$.

Proposition 4.2. Let $n$ be an odd integer. The elements of $\mathcal{P} \mathcal{O I}_{n} \cap J_{n-1}$ corresponding to canonical words of the form $x_{i} \cdots x_{n-1} x_{0} x_{1} \cdots x_{n-i}$, with $1 \leq$ $i \leq n-1$, are in the abelian kernel of $\mathcal{P O D} \mathcal{I}_{n}$.

Proof. Let $p \in \mathcal{P O D} \mathcal{I}_{n}$ be an element corresponding to a canonical word $w$ as in the statement of the proposition. Let $v \in \varphi^{-1}(p)$ be a word such that $|v|_{x_{i}}=k$, for $0 \leq i \leq n-1$. Such a word exists by the discussion above. The last component of $\gamma(v)$ is an even integer corresponding to the $h^{2}$ 's introduced using the relation $N R_{0}$. We then conclude that $(k, k, \ldots, k, \ell)+$ 
$(1, \ldots, 1,0) \mathbb{N}+(0, \ldots, 0,2) \mathbb{N} \subseteq \gamma\left(\varphi^{-1}(p)\right)$ since the relations $R_{5}$ and $N R_{0}$ can be used any number of times. Thus $(0, \ldots, 0) \in(k, k, \ldots, k, \ell)+(1, \ldots, 1,0) \mathbb{Z}+$ $(0, \ldots, 0,2) \mathbb{Z} \subseteq \overline{\gamma\left(\varphi^{-1}(p)\right)}$, as required.

Next we complete the computation of the abelian kernel of $\mathcal{P O D} \mathcal{I}_{n}$. We prove that the elements of $\mathcal{P O D \mathcal { I } _ { n }}$ belonging to $\mathrm{K}_{\mathrm{Ab}}\left(\mathcal{P O D} \mathcal{I}_{n}\right) \backslash \mathrm{K}_{\mathrm{Ab}}\left(\mathcal{P O} \mathcal{I}_{n}\right)$ are exactly those given in the previous proposition when $n$ is odd and that there are no such elements when $n$ is even. In particular, for even $n$, the equality $\mathrm{K}_{\mathrm{Ab}}\left(\mathcal{P} \mathcal{O I}_{n}\right)=\mathrm{K}_{\mathrm{Ab}}\left(\mathcal{P} \mathcal{O} \mathcal{D} \mathcal{I}_{n}\right) \cap \mathcal{P O} \mathcal{I}_{n}$ happens.

We say that $u \in Y^{*}$ is a soft word if whenever $x_{i} h^{k} x_{j}$, with $k \in \mathbb{N}$ and $0 \leq i, j \leq n-1$, is a factor of $u$, then

$$
j \equiv \begin{cases}(n-i) \bmod n & \text { if } k \text { is odd } \\ (i+1) \bmod n & \text { if } k \text { is even. }\end{cases}
$$

Note 1. Observe that a word $x_{i} x_{j}$, with $0 \leq i, j \leq n-1$, is soft if and only if $(i, j)=(n-1,0)$ or $j=i+1$, for $i<n-1$. Therefore, $R_{1} \cup R_{2} \cup R_{3} \cup$ $R_{4} \cup R_{6} \cup N R_{2}$ cannot be applied to soft words, since both sides of each of these relations contain as a factor a non soft word of length two.

It is clear that words of the form $x_{i+1} \cdots x_{n-1} x_{0} x_{1} \cdots x_{j}$ and $x_{i+1} \cdots$ $x_{n-1} x_{0} x_{1} \cdots x_{j} h$ are soft. Thus, all elements of the $\mathscr{J}$-class $J_{n-1}$ of $\mathcal{P O D} \mathcal{I}_{n}$ may be represented through soft words. In fact, any word representing one such element must be soft, as follows from the note above, the next lemma and the fact that $\langle Y \mid R\rangle$ is a presentation of $\mathcal{P O D} \mathcal{I}_{n}$.

Lemma 4.3. Each word of $Y^{*}$ connected by an elementary $\left(R_{5} \cup N R_{0} \cup\right.$ $\left.N R_{1}\right)$-transition to a soft word $Y^{*}$ is a soft word too.

Proof. Let $u=v$ be a relation from $R_{5} \cup N R_{0} \cup N R_{1}$ and $a, b \in Y^{*}$. Then, it suffices to prove that $a u b$ is soft if and only if $a v b$ is soft. This is clear if $u=v$ belongs to $R_{5} \cup N R_{0}$, whence we may suppose that $u=v$ comes from $N R_{1}$. Then, $u=h x_{j}$, for some $j \in\{0,1, \ldots, n-1\}$.

Let $a^{\prime}$ be a word of $Y^{*}$ such that $a=a^{\prime} h^{k_{1}}$, for some $k_{1} \geq 0$, and $a^{\prime}$ does not admit $h$ as a suffix. Let $b^{\prime}$ be a word of $Y^{*}$ such that $b=h^{k_{2}} b^{\prime}$, for some $k_{2} \geq 0$, and $b^{\prime}$ does not admit $h$ as a prefix. Say that (s) is satisfied if $a^{\prime}$ is the empty word or $a^{\prime}=a^{\prime \prime} x_{i}$, with $a^{\prime \prime} \in Y^{*}$ and $i \in\{0,1, \ldots, n-1\}$ such that

$$
i \equiv \begin{cases}(n-j) \bmod n & \text { if } k_{1} \text { is even } \\ (j-1) \bmod n & \text { if } k_{1} \text { is odd }\end{cases}
$$

and if $b^{\prime}$ is the empty word or $b^{\prime}=x_{\ell} b^{\prime \prime}$, with $b^{\prime \prime} \in Y^{*}$ and $\ell \in\{0,1, \ldots, n-1\}$ such that

$$
\ell \equiv \begin{cases}(n-j) \bmod n & \text { if } k_{2} \text { is odd } \\ (j+1) \bmod n & \text { if } k_{2} \text { is even }\end{cases}
$$

Then, it is a routine matter to show that $a w b$ is soft if and only if (s) is satisfied, for $w \in\{u, v\}$. Thus, $a u b$ is soft if and only if $a v b$ is soft, as required. 
Successive applications of transitions from $R_{5} \cup N R_{0} \cup N R_{1}$ show that, for any $x \in \mathcal{P O D} \mathcal{I}_{n}$,

$$
\begin{aligned}
& \gamma\left(w_{x}\right)+(1,1, \ldots, 1,0) \mathbb{Z}+(0,0, \ldots, 0,2) \mathbb{Z} \\
& +\left(1,0_{(2)}, 1, \ldots, 1,0_{(n-1)}, 0\right) \mathbb{Z}+\left(1,1,0_{(3)}, 1, \ldots, 1,0_{(n-2)}, 1,0\right) \mathbb{Z} \\
& +\cdots\left[+\left(1, \ldots, 1,-1_{(n / 2+1)}, 1, \ldots, 1,0\right) \mathbb{Z}\right]+(-1,1,1, \ldots, 1,0) \mathbb{Z} \subseteq \overline{\gamma\left(\varphi^{-1}(x)\right)},
\end{aligned}
$$

where $w_{x}$ is any word corresponding to $x$ and the part in square brackets only appears in case $n$ is even.

By the comments preceding Lemma 4.3 it follows that, when $x \in J_{n-1}$, the inclusion above is in fact an equality. Thus an element $x \in J_{n-1}$, corresponding to a word $w_{x}$ belongs to the abelian kernel of $\mathcal{P O D \mathcal { I } _ { n }}$ if and only if $\gamma\left(w_{x}\right) \in(1,1, \ldots, 1,0) \mathbb{Z}+(0, \ldots, 0,2) \mathbb{Z}+\left(0,1_{(2)}, 0, \ldots, 0,1_{(n-1)}, 0 \mathbb{Z}+\right.$ $\cdots\left[+\left(0,0, \ldots, 0,2_{(n / 2+1)}, 0, \ldots, 0\right) \mathbb{Z}\right]+(2,0,0, \ldots, 0) \mathbb{Z}$.

Suppose now that $w_{x}=x_{i+1} \cdots x_{n-1} x_{0} x_{1} \cdots x_{j}$ is the canonical word associated to $x$. It is clear that $x \in \mathrm{K}_{\mathrm{Ab}}\left(\mathcal{P} \mathcal{O} \mathcal{D} \mathcal{I}_{n}\right)$ implies that $\left|w_{x}\right|_{x_{r}}=$ $\left|w_{x}\right|_{x_{n-r}}(1 \leq r \leq n-1)$.

Corollary 4.4. If $n$ is an odd integer, then the abelian kernel of $\mathcal{P O D} \mathcal{I}_{n}$ consists of the abelian kernel of $\mathcal{P O I}_{n}$ united with the set of elements corresponding to words of the form $x_{i} \cdots x_{n-1} x_{0} x_{1} \cdots x_{n-i}$, for $1 \leq i \leq n-1$.

It is also clear that, when $n$ is even, if $x \in \mathrm{K}_{\mathrm{Ab}}\left(\mathcal{P O} \mathcal{O} \mathcal{I}_{n}\right)$, then the first and the $(n / 2+1)^{t h}$ components of $\gamma\left(w_{x}\right)$ must have the same parity. Checking the forms of the canonical words corresponding to elements in $J_{n-1}$ we easily obtain that

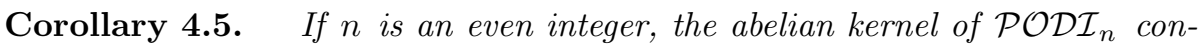
sists precisely of the elements of the abelian kernel of $\mathcal{P O} \mathcal{I}_{n}$.

The following pictures represent possible drawings of the egg-boxes of the $\mathscr{J}$-classes $J_{n-1}$ for $n=4$ and $n=5$, respectively. Only the elements of the abelian kernel are marked: the idempotents are represented by the symbol * and the non-idempotents by the symbol + .

The picture for $n=4$ illustrates the general case for $n$ even and the picture for $n=5$ illustrates the general case for $n$ odd.

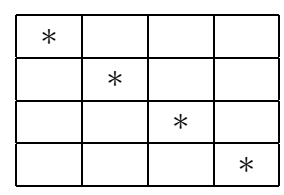

\begin{tabular}{|c|c|c|c|c|}
\hline$*$ & & & & + \\
\hline & $*$ & & + & \\
\hline & & $*$ & & \\
\hline & + & & $*$ & \\
\hline+ & & & & $*$ \\
\hline
\end{tabular}

\section{The abelian kernel of $\mathcal{P O} \mathcal{O} \mathcal{I}_{n}$}

Recall that the $\mathscr{J}$-class $J_{n}$ of $\mathcal{P O} \mathcal{R} \mathcal{I}_{n}$ is the dihedral group generated by $h$ (order 2) and $g$ (order $n$ ). Using Lemma 2.8, we have that $\mathrm{K}_{\mathrm{Ab}}\left(\mathcal{P} \mathcal{O} \mathcal{R} \mathcal{I}_{n}\right) \cap J_{n}=$ 
$J_{n}^{\prime}$. Since the derived subgroup $J_{n}^{\prime}$ is $\left\langle g^{2}\right\rangle$, we have that $\mathrm{K}_{\mathrm{Ab}}\left(\mathcal{P O} \mathcal{R} \mathcal{I}_{n}\right) \cap J_{n}$ consists of the even powers of $g$ when $n$ is even, and contains $g$ when $n$ is odd.

Analogously to the case of $\mathcal{P O D} \mathcal{I}_{n}$, now using Corollary 3.6 and Theorem 2.7, one has that, for $k<n-1, \mathrm{~K}_{\mathrm{Ab}}\left(\mathcal{P O} \mathcal{R} \mathcal{I}_{n}\right) \cap J_{k}$ consists of the elements of $J_{k}$ that belong to $\mathcal{P O P \mathcal { I } _ { n }}$. To obtain the complete description of the abelian kernel of $\mathcal{P O R \mathcal { I } _ { n }}$ it remains to decide which non idempotents of $\mathcal{P O P \mathcal { I } _ { n }} \cap J_{n-1}$ belong to $\mathrm{K}_{\mathrm{Ab}}\left(\mathcal{P O R} \mathcal{I}_{n}\right)$. Again the parity of $n$ has a great influence.

We begin by stating a couple of lemmas whose proofs depend just on the observation of the relations $\tilde{R}$, which (as noticed above) are the only relations applicable to words representing elements of $J_{n-1}$.

Lemma 5.1. If $n$ is even and $w$ is a word obtained from another word $u$ through the application of a relation of $\tilde{R}$, then the lengths $|u|$ and $|w|$ have the same parity.

Lemma 5.2. If $n$ is odd and $w$ is a word obtained from another word $u$ through the application of a relation of $\tilde{R}$ and $B=\left\{x_{1}, \ldots, x_{n-1}\right\}$, then $|u|_{B}$ and $|w|_{B}$ have the same parity.

Our next lemma establishes a set of relations holding in $\mathcal{P O R} \mathcal{I}_{n}$ that we will use in the sequel.

Lemma 5.3. For $1 \leq k \leq n-1$, the equality $\left(g x_{n-1}\right)^{k}=g^{k} x_{n-k} x_{n-(k-1)}$ $\cdots x_{n-1}$ holds in $\mathcal{P O R} \mathcal{I}_{n}$.

Proof. We prove the equality by induction on $k$. For $k=1$ the equality is trivial. Suppose now that $1 \leq k<n-1$. Using the induction hypothesis and $R_{7}$, we have

$$
\left(g x_{n-1}\right)^{k+1}=g^{k} x_{n-k} x_{n-(k-1)} \cdots x_{n-1} \cdot g x_{n-1}=g^{k+1} x_{n-(k+1)} x_{n-k} \cdots x_{n-1},
$$

as required.

Now, since the $(n-1)$-power of the element $g x_{n-1}$ (of the $\mathscr{J}$-class $J_{n-1}$ of $\left.\mathcal{P O R} \mathcal{I}_{n}\right)$ is $g^{n-1} x_{1} \cdots x_{n-1}$, which is an idempotent, and any other power $\left(g x_{n-1}\right)^{k}$, with $1 \leq k<n-1$, is equal by the previous lemma and the relation $R_{5}$ to $g^{k} x_{n-k} x_{n-(k-1)} \cdots x_{n-1} x_{0} x_{1} \cdots x_{n-1}$, which is non-idempotent (see Section 1), we have:

Proposition 5.4. The subsemigroup $\left\langle g x_{n-1}\right\rangle$ of $\mathcal{P O R} \mathcal{I}_{n}$ is a group of order $n-1$ contained in the $\mathscr{J}$-class $J_{n-1}$.

Let $H$ be the maximal subgroup of $J_{n-1} \cap \mathcal{P O} \mathcal{R} \mathcal{I}_{n}$ containing $g x_{n-1}$. Recall that it is a dihedral group of order $2(n-1)$, whence it is generated by the element $g x_{n-1}$ of order $n$ and an element $h^{\prime} \notin\left\langle g x_{n-1}\right\rangle$ of order 2 . So, the derived subgroup $H^{\prime}$ is the cyclic subgroup $\left\langle\left(g x_{n-1}\right)^{2}\right\rangle$. It is $\left\langle g x_{n-1}\right\rangle$ when $n-1$ is odd (i.e. $n$ is even) and consists of the elements 
$g^{2} x_{n-2} x_{n-1}, g^{4} x_{n-4} x_{n-3} x_{n-2} x_{n-1}, \ldots, g^{n-1} x_{1} x_{2} \cdots x_{n-2} x_{n-1}=x_{0} x_{1} \cdots x_{n-1}$ when $n$ is odd.

Thus, we have proved the following lemmas which imply that if $n$ is even, then half of the elements of $H$ belong to $\mathrm{K}_{\mathrm{Ab}}\left(\mathcal{P} \mathcal{O} \mathcal{R} \mathcal{I}_{n}\right)$, while if $n$ is odd, only a quarter of the elements of $H$ belong to $\mathrm{K}_{\mathrm{Ab}}\left(\mathcal{P} \mathcal{O} \mathcal{R} \mathcal{I}_{n}\right)$.

Lemma 5.5. If $n$ is even, then the elements corresponding to words of the form $\left(g x_{n-1}\right)^{k}$, with $k \geq 1$, belong to $\mathrm{K}_{\mathrm{Ab}}\left(\mathcal{P O O R} \mathcal{I}_{n}\right) \cap J_{n-1}$.

Lemma 5.6. If $n$ is odd, then the elements corresponding to words of the form $\left(g x_{n-1}\right)^{k}$, where $k \geq 2$ is an even integer, belong to $\mathrm{K}_{\mathrm{Ab}}\left(\mathcal{P} \mathcal{O} \mathcal{R} \mathcal{I}_{n}\right) \cap J_{n-1}$.

Next, we state a technical lemma which in fact gives us a new set of representative words of the elements of the $\mathscr{J}$-class $J_{n-1}$ of $\mathcal{P O P} \mathcal{I}_{n}$.

Lemma 5.7. One has $g^{k}\left(x_{n-i} \cdots x_{n-1}\right) x_{0}\left(x_{1} \cdots x_{j}\right)=g^{2 n+k-i-j-1} g^{j+1}$ $\left(g x_{n-1}\right)^{i+j} g^{n-j-1}$, for all $1 \leq i \leq n-1,0 \leq j \leq n-1$ and $0 \leq k \leq n-1$.

Proof. First, we observe that $x_{1} g=g^{2} x_{n-1} x_{0}$ (see [9, Lemma 5.4]).

Next, we prove the equality

$$
x_{n-1} x_{0}\left(x_{1} \cdots x_{j}\right) g^{j+1}=x_{n-1}\left(g x_{n-1}\right)^{j}, \quad 0 \leq j \leq n-1 \text {, }
$$

by induction on $j$. For $j=0$ the equality is one of the relations $R_{8}$. Then, let $0 \leq j<n-1$. Using $R_{7}$, the relation above, $R_{8}$ and the induction hypothesis (by this order), we have

$$
\begin{aligned}
x_{n-1} x_{0}\left(x_{1} \cdots x_{j+1}\right) g^{j+2} & =x_{n-1} x_{0} x_{1}\left(x_{2} \cdots x_{j+1}\right) g g^{j+1} \\
& =x_{n-1} x_{0} \cdot x_{1} g \cdot\left(x_{1} \cdots x_{j}\right) g^{j+1} \\
& =x_{n-1} x_{0} g^{2} x_{n-1} x_{0}\left(x_{1} \cdots x_{j}\right) g^{j+1} \\
& =x_{n-1} g \cdot x_{n-1} x_{0}\left(x_{1} \cdots x_{j}\right) g^{j+1} \\
& =x_{n-1} g x_{n-1}\left(g x_{n-1}\right)^{j} \\
& =x_{n-1}\left(g x_{n-1}\right)^{j+1} .
\end{aligned}
$$

Now, using equality (1), $R_{9}$ and Lemma 5.3 , we obtain

$$
\begin{aligned}
g^{k}\left(x_{n-i} \cdots x_{n-1}\right) x_{0}\left(x_{1} \cdots x_{j}\right) & =g^{k}\left(x_{n-i} \cdots x_{n-2}\right) x_{n-1} x_{0}\left(x_{1} \cdots x_{j}\right) g^{j+1} g^{n-j-1} \\
& =g^{n+k-i} g^{i} x_{n-i} \cdots x_{n-2} x_{n-1}\left(g x_{n-1}\right)^{j} g^{n-j-1} \\
& =g^{n+k-i}\left(g x_{n-1}\right)^{i}\left(g x_{n-1}\right)^{j} g^{n-j-1} \\
& =g^{2 n+k-i-j-1} g^{j+1}\left(g x_{n-1}\right)^{i+j} g^{n-j-1}
\end{aligned}
$$

as required.

Now, we can describe the elements of $J_{n-1}$ that belong to the abelian kernel of $\mathcal{P O R \mathcal { I } _ { n }}$. 
Theorem 5.8. Let $B=\left\{x_{1}, \ldots, x_{n-1}\right\}$ and let $x$ be the element of $J_{n-1} \cap$ $\mathcal{P O P} \mathcal{I}_{n}$ corresponding to the word $w=g^{k}\left(x_{n-i} \cdots x_{n-1}\right) x_{0}\left(x_{1} \cdots x_{j}\right)$, with $0 \leq k \leq n-1,1 \leq i \leq n-1$ and $0 \leq j \leq n-1$. Then $x \in \mathrm{K}_{\mathrm{Ab}}\left(\mathcal{P O} \mathcal{R} \mathcal{I}_{n}\right)$ if and only if:

(i) $|w|_{B}$ is even, for $n$ odd;

(ii) $|w|$ is even, for $n$ even.

Proof. First, we prove (i). Let us suppose that $n$ is odd. If $x \in$ $\mathrm{K}_{\mathrm{Ab}}\left(\mathcal{P O} \mathcal{R} \mathcal{I}_{n}\right)$ then, by Corollary 3.3 , there exists a word $u$ representing $x$ such that $|u|_{B}$ is even. Hence, since $w$ is obtained from $u$ through the application of relations from $\tilde{R}$, by Lemma $5.2,|w|_{B}$ must also be even.

Conversely, admit that $|w|_{B}$ is even. As $|w|_{B}=i+j$, by Lemma 5.6, $\left(g x_{n-1}\right)^{i+j} \in \mathrm{K}_{\mathrm{Ab}}\left(\mathcal{P O R} \mathcal{I}_{n}\right)$, whence $g^{j+1}\left(g x_{n-1}\right)^{i+j} g^{n-j-1} \in \mathrm{K}_{\mathrm{Ab}}\left(\mathcal{P O R} \mathcal{I}_{n}\right)$, as $\left(g^{j+1}, g^{n-j-1}\right)$ is a conjugating pair. Finally, as we notice at the beginning of this section, $g \in \mathrm{K}_{\mathrm{Ab}}\left(\mathcal{P O R} \mathcal{I}_{n}\right) \cap J_{n}$ and so, by Lemma 5.7, we deduce that $x \in \mathrm{K}_{\mathrm{Ab}}\left(\mathcal{P O R} \mathcal{I}_{n}\right)$.

The proof of (ii) is quite similar to the previous one. In fact, if $x \in$ $\mathrm{K}_{\mathrm{Ab}}\left(\mathcal{P O} \mathcal{R} \mathcal{I}_{n}\right)$ then, by Corollary 3.3 , there exists a word $u$ representing $x$ such that $|u|$ is even. Hence, since $w$ is obtained from $u$ through the application of just relations from $\tilde{R}$, by Lemma 5.1, $|w|$ also is even.

On the other hand, suppose that $|w|$ is even. Then, by Lemma 5.5, we have $\left(g x_{n-1}\right)^{i+j} \in \mathrm{K}_{\mathrm{Ab}}\left(\mathcal{P O} \mathcal{R} \mathcal{I}_{n}\right)$ and so $g^{j+1}\left(g x_{n-1}\right)^{i+j} g^{n-j-1} \in \mathrm{K}_{\mathrm{Ab}}$ $\left(\mathcal{P O R} \mathcal{I}_{n}\right)$. Now, by Lemma 5.7 , it suffices to show that $g^{2 n+k-i-j-1} \in$ $\mathrm{K}_{\mathrm{Ab}}\left(\mathcal{P O} \mathcal{R} \mathcal{I}_{n}\right)$. In order to show it, observe that, since $k+i+j+1=|w|$ is even, then $k-i-j-1$ is even and so it is $2 n+k-i-j-1$, whence $g^{2 n+k-i-j-1} \in \mathrm{K}_{\mathrm{Ab}}\left(\mathcal{P O} \mathcal{R} \mathcal{I}_{n}\right) \cap J_{n}$, as noticed at the beginning of this section.

We thus conclude that half of the elements of $J_{n-1} \cap \mathcal{P O P} \mathcal{I}_{n}$ belong the abelian kernel of $\mathcal{P O R} \mathcal{I}_{n}$. An analysis on its distribution into $\mathscr{H}$-classes leads to the egg-boxes illustrated in the following pictures. They represent possible drawings of the egg-boxes for $n=4$ and $n=5$, respectively. The number inscribed in a box corresponding to a $\mathscr{H}$-class represents the number of elements of the $\mathscr{H}$-class which are in the abelian kernel compared to the total number of elements of the $\mathscr{H}$-class.

The picture for $n=4$ illustrates the general case for $n$ even and the picture for $n=5$ illustrates the general case for $n$ odd.

\begin{tabular}{|c|c|c|c|}
\hline$\frac{1}{2}$ & & $\frac{1}{2}$ & \\
\hline & $\frac{1}{2}$ & & $\frac{1}{2}$ \\
\hline$\frac{1}{2}$ & & $\frac{1}{2}$ & \\
\hline & $\frac{1}{2}$ & & $\frac{1}{2}$ \\
\hline
\end{tabular}

\begin{tabular}{|c|c|c|c|c|}
\hline$\frac{1}{4}$ & $\frac{1}{4}$ & $\frac{1}{4}$ & $\frac{1}{4}$ & $\frac{1}{4}$ \\
\hline$\frac{1}{4}$ & $\frac{1}{4}$ & $\frac{1}{4}$ & $\frac{1}{4}$ & $\frac{1}{4}$ \\
\hline$\frac{1}{4}$ & $\frac{1}{4}$ & $\frac{1}{4}$ & $\frac{1}{4}$ & $\frac{1}{4}$ \\
\hline$\frac{1}{4}$ & $\frac{1}{4}$ & $\frac{1}{4}$ & $\frac{1}{4}$ & $\frac{1}{4}$ \\
\hline$\frac{1}{4}$ & $\frac{1}{4}$ & $\frac{1}{4}$ & $\frac{1}{4}$ & $\frac{1}{4}$ \\
\hline
\end{tabular}


We have to remark that a short argument (making use of Lemmas 5.1 and 5.2) like the one used to prove that some elements do not belong to the abelian kernel of $\mathcal{P O R} \mathcal{I}_{n}$ does not work for $\mathcal{P O D} \mathcal{I}_{n}$. If one wished to make a similar proof, more cases would have to be treated and it would produce a proof possibly longer than the one we gave.

\section{The abelian kernel of $\mathcal{I}_{n}$}

The $\mathscr{J}$-class $J_{n}$ of $\mathcal{I}_{n}$ is precisely the symmetric group $\mathcal{S}_{n}$, therefore its derived subgroup is the alternating group $\mathcal{A}_{n}$ (see [7]). From Lemma 2.8 it follows that $\mathrm{K}_{\mathrm{Ab}}\left(\mathcal{I}_{n}\right) \cap J_{n}=\mathcal{A}_{n}$.

Next we will consider the elements of the $\mathscr{J}$-class $J_{n-1}$.

Let $1 \leq r, s \leq n$. We denote by $H_{s}^{r}$ the $\mathscr{H}$-class of $J_{n-1}$ of the elements $x$ such that $\operatorname{Dom}(x)=\{1,2, \ldots, n\} \backslash\{r\}$ and $\operatorname{Im}(x)=\{1,2, \ldots, n\} \backslash\{s\}$. We define $K_{s}^{r}=H_{s}^{r} \cap \mathrm{K}_{\mathrm{Ab}}\left(\mathcal{I}_{n}\right)$.

Lemma 6.1. For each $1 \leq r, s, u, v \leq n$, there exist $\sigma, \nu \in \mathcal{A}_{n}$ such that $\sigma K_{s}^{r} \nu=K_{v}^{u}$ and $\sigma K_{v}^{u} \nu=K_{s}^{r}$.

Proof. Define $\sigma$ as the identity if $r=u$ or, otherwise, $\sigma=(i j)(r u)$, with $1 \leq i, j \leq n$ any two integers such that $i, j, r, u$ are four distinct integers (notice that $n \geq 4)$. Also, define $\nu$ as the identity if $s=v$ or, otherwise, $\nu=(s v)(k \ell)$, with $1 \leq k, \ell \leq n$ any two integers such that $k, l, s, v$ are four distinct integers. Then, given $x \in H_{s}^{r}$ (respectively, $x \in H_{v}^{u}$ ), it is a routine matter to show that $\sigma x \nu \in H_{v}^{u}$ (respectively, $\sigma x \nu \in H_{s}^{r}$ ). In particular, $\sigma K_{s}^{r} \nu \subseteq H_{v}^{u}$ and $\sigma K_{v}^{u} \nu \subseteq H_{s}^{r}$. Furthermore, since $\sigma, \nu \in \mathcal{A}_{n} \subseteq \mathrm{K}_{\mathrm{Ab}}\left(\mathcal{I}_{n}\right)$, then $\sigma K_{s}^{r} \nu \subseteq K_{v}^{u}$ and $\sigma K_{v}^{u} \nu \subseteq K_{s}^{r}$. Now, as $\sigma$ and $\nu$ are permutations of order one or two, we have $K_{s}^{r}=\sigma \sigma K_{s}^{r} \nu \nu \subseteq \sigma K_{v}^{u} \nu \subseteq K_{s}^{r}$, whence $K_{s}^{r}=\sigma K_{v}^{u} \nu$. Similarly, we also have $K_{v}^{u}=\sigma K_{s}^{r} \nu$, as required.

It follows immediately from this result that each $\mathscr{H}$-class of $\mathcal{I}_{n}$ contained in $J_{n-1}$ has the same number of elements belonging to the abelian kernel of $\mathcal{I}_{n}$.

The next lemma gives an example of an element of $J_{n-1}$ that does not belong to the abelian kernel of $\mathcal{I}_{n}$.

Lemma 6.2. The element of $J_{n-1}$ represented by ac does not belong to the abelian kernel of $\mathcal{I}_{n}$.

Proof. Note that the words appearing in the relations $(c a)^{2}=c a c=(a c)^{2}$ correspond to an element of rank $n-2$, whence they can not be applied to any word representing the same element than $a c$ does.

If $n$ is odd then any word appearing in the remaining relations has an even number of $a$ 's. Hence, by applying these relations to $a c$, one only gets words with an odd number of $a$ 's. The result follows by Theorem 3.2.

The proof is analogous if $n$ is even. In fact, in this case, observe that, in the words appearing in the same relations, the sum of the number of $a$ 's with 
the number of $g$ 's is even. Then, using Corollary 3.3 with $B=\{a, g\}$, one gets the result.

Let $H$ be a maximal subgroup of $J_{n-1}$. It is isomorphic to the symmetric group $\mathcal{S}_{n-1}$, whence its derived subgroup is isomorphic to the alternating group $\mathcal{A}_{n-1}$. Thus $H^{\prime}$ is a subgroup of $H$ of index 2 , and so a subgroup of $H$ strictly containing $H^{\prime}$ must be $H$ itself. Since $\mathrm{K}_{\mathrm{Ab}}\left(\mathcal{I}_{n}\right) \cap H$ is a subgroup of $H$ containing $H^{\prime}$ it is either $H$ or $H^{\prime}$.

Now, since by Lemma 6.2 there exists one element of $J_{n-1}$ that does not belong to $\mathrm{K}_{\mathrm{Ab}}\left(\mathcal{I}_{n}\right)$ and, as observed above, from Lemma 6.1 it follows that the number of elements in each $\mathscr{H}$-class of $J_{n-1}$ that belong to $\mathrm{K}_{\mathrm{Ab}}\left(\mathcal{I}_{n}\right)$ is the same, then each $\mathscr{H}$-class of $J_{n-1}$ has precisely half of its elements in the abelian kernel of $\mathcal{I}_{n}$. Therefore:

Proposition 6.3. $\quad$ Let $H$ be a maximal subgroup of the $\mathscr{J}$-class $J_{n-1}$. Then $\mathrm{K}_{\mathrm{Ab}}\left(\mathcal{I}_{n}\right) \cap H=H^{\prime}$. Moreover, one has $\left|J_{n-1} \cap \mathrm{K}_{\mathrm{Ab}}\left(\mathcal{I}_{n}\right)\right|=\frac{1}{2}\left|J_{n-1}\right|$.

Furthermore, we have the following description of the elements of $J_{n-1}$ that belong to $\mathrm{K}_{\mathrm{Ab}}\left(\mathcal{I}_{n}\right)$ :

Proposition 6.4. $\quad$ Let $H$ be any maximal subgroup of $J_{n-1}$. Then

$$
J_{n-1} \cap \mathrm{K}_{\mathrm{Ab}}\left(\mathcal{I}_{n}\right)=\mathcal{A}_{n-1} H^{\prime} \mathcal{A}_{n-1} .
$$

Proof. From Lemmas 6.1 and 6.3 it follows that $J_{n-1} \cap \mathrm{K}_{\mathrm{Ab}}\left(\mathcal{I}_{n}\right) \subseteq \mathcal{A}_{n-1} H^{\prime}$ $\mathcal{A}_{n-1}$. The converse follows immediately from the fact that both $H^{\prime}$ and $\mathcal{A}_{n-1}$ are contained in $\mathrm{K}_{\mathrm{Ab}}\left(\mathcal{I}_{n}\right)$ and $\mathcal{A}_{n-1} H^{\prime} \mathcal{A}_{n-1}$ only contains maps of rank $n-1$.

It remains to determine the elements in the $\mathscr{J}$-classes $J_{k}$, with $0 \leq k<$ $n-1$, that belong to $\mathrm{K}_{\mathrm{Ab}}\left(\mathcal{I}_{n}\right)$. We will easily show that they all are.

Let $0 \leq k \leq n-2$ and let $x$ be an element of $J_{k}$. Denote, as usual, by $x^{-1}$ the inverse (in $\mathcal{I}_{n}$ ) of $x$. Let $\sigma$ be any permutation of $\mathcal{S}_{n}$ that extends $x$, let $1 \leq i, j \leq n$ be any two distinct integers that do not belong to the image of $x$ and let $\nu$ be the transposition $(i j)$. Clearly, $x=x x^{-1} \sigma=x x^{-1} \sigma \nu$. Since $x x^{-1}$ is an idempotent, it belongs to $\mathrm{K}_{\mathrm{Ab}}\left(\mathcal{I}_{n}\right)$. On the other hand, we have $\sigma \in \mathcal{A}_{n}$ or $\sigma \nu \in \mathcal{A}_{n}$, whence $x \in \mathrm{K}_{\mathrm{Ab}}\left(\mathcal{I}_{n}\right)$. Hence, we have proved:

Proposition 6.5. $\quad$ All elements of $\mathcal{I}_{n}$ with rank less than $n-1$ belong to $\mathrm{K}_{\mathrm{Ab}}\left(\mathcal{I}_{n}\right)$.

Finally, in short, we can state the following result:

Theorem 6.6. The abelian kernel of $\mathcal{I}_{n}$ consists of all even permutations of $\mathcal{S}_{n}$, of all the $\frac{1}{2}\left|J_{n-1}\right|$ elements (with rank $n-1$ ) of $\mathcal{A}_{n-1} H^{\prime} \mathcal{A}_{n-1}$, where $H$ is any maximal subgroup of $J_{n-1}$, and of all transformations with rank less than $n-1$. 


\section{References}

[1] Almeida, J., "Finite Semigroups and Universal Algebra", World Scientific, Singapore, 1995. English translation.

[2] Ash, C.J., Inevitable graphs: a proof of the type II conjecture and some related decision procedures, Int. J. Algebra Comput. 1 (1991) 127-146.

[3] Catarino, P.M., and P.M. Higgins, The monoid of orientation-preserving mappings on a chain, Semigroup Forum 58 (1999) 190-206.

[4] Delgado, M., Abelian pointlikes of a monoid, Semigroup Forum 56 (1998) $339-361$.

[5] Delgado, M., Computing commutative images of rational languages and applications, Theor. Inform. Appl. 35 (2001) 419-435.

[6] Delgado, M., and V.H. Fernandes, Abelian kernels of some monoids of injective partial transformations and an application, Semigroup Forum 61 (2000) 435-452.

[7] Dummit, M.S., and R.M. Foote, "Abstract Algebra", Prentice-Hall, Englewood Clifs, N.J., 1991.

[8] Fernandes, V.H., Semigroups of order-preserving mappings on a finite chain: a new class of divisors, Semigroup Forum 54 (1997) 230-236.

[9] Fernandes, V.H., The monoid of all injective orientation-preserving partial transformations on a finite chain, Comm. Algebra 28 (2000) 3401-3426.

[10] Fernandes, V.H., The monoid of all injective order-preserving partial transformations on a finite chain, Semigroup Forum 62 (2001) 178-204.

[11] Fernandes, V.H., Presentations for some monoids of partial transformations on a finite chain: a survey, in "Semigroups, Algorithms, Automata and Languages", G.M.S. Gomes, J.-E. Pin, and P.V. Silva (eds.), World Scientific, 2002, 363-378.

[12] Fernandes, V.H., G.M.S. Gomes, and M.M. Jesus, Presentations for some monoids of injective partial transformations on a finite chain, Southeast Asian Bull. Math. (to appear).

[13] Henckell, K., S. Margolis, J.-E. Pin, and J. Rhodes, Ash's type II theorem, profinite topology and Malcev products. Part I, Int. J. Algebra Comput. 1 (1991) 411-436.

[14] Howie, J.M., "Fundamentals of Semigroup Theory", Oxford University Press, 1995.

[15] McAlister, D., Semigroups generated by a group and an idempotent, Comm. Algebra 26 (1998) 515-547. 
[16] Moore, E.H., Concerning the abstract groups of order $k$ ! and $\frac{1}{2} k$ ! holohedrically isomorphic with the symmetric and the alternating substitution groups on $k$ letters, Proc. London Math. Soc. 28(1) (1897) 357-366.

[17] Pin, J.-E., "Varieties of Formal Languages", Plenum, London, 1986. English translation.

[18] Popova, L.M., The defining relations of certain semigroups of partial transformations of a finite set, Leningradskij gosudarstvennyj pedagogicheskij institut imeni A. I. Gerzena, Uchenye Zapiski 218 (1961) 191-212 (in Russian).

[19] Ribes, L., and P.A. Zalesskiı̌, On the profinite topology on a free group, Bull. London Math. Soc. 25 (1993) 37-43.

[20] Tilson, B., Type II redux, in "Semigroups and Their Applications", S. M. Goberstein and P. M. Higgins (eds.), Reidel, Dordrecht, 1987, 201-205.

[21] The GAP Group, GAP - Groups, Algorithms, and Programming, Version 4.3, 2002. (http://www.gap-system.org).

Manuel Delgado

Centro de Matemática

Universidade do Porto

Rua do Campo Alegre, 687

4169-007 Porto, Portugal

mdelgado@fc.up.pt
Vítor H. Fernandes

Departamento de Matemática

Faculdade de Ciencias e Tecnologia

Universidade Nova de Lisboa

Monte da Caparica

2829-516 Caparica, Portugal

and

C.A.U.L.

1649-003 Lisboa, Portugal

vhf@mail.fct.unl.pt

Received July 29, 2003

and in final form September 10, 2003

Online publication January 12, 2004 